\title{
APPLICATION OF DIGITAL SIGNAL PROCESSING AND MACHINE LEARNING FOR ELECTROMYOGRAPHY: A REVIEW
}

\author{
S.N. Omar ${ }^{1}$, N.M. Saad ${ }^{1,}{ }^{,}$, A.R. Abdullah ${ }^{2}$, E.F. Shair ${ }^{2}$ and T.N.S.T. \\ Zawawi $^{2}$ \\ ${ }^{1}$ Faculty of Electrical and Electronic Engineering Technology. \\ ${ }^{2}$ Faculty of Electrical Engineering. \\ Universiti Teknikal Malaysia Melaka, Durian Tunggal, 76100, Melaka, Malaysia \\ "Corresponding Author's Email: ${ }^{1}$ norhashimah@utem.edu.my \\ Article History: Received June 7, 2021; Revised July 9, 2021; \\ Accepted July 16, 2021
}

\begin{abstract}
Digital signal processing (DSP) and Machine learning (ML) have emerged as promising approaches to automate prediction tasks into electromyography (EMG) muscles conditions. To fill the research gap, This paper reviews the state-of-the-art applications of DSP and ML for EMG signal analysis. DSP techniques to extract information of EMG signal is highly needed. The major disadvantage of the frequency domain approach is it does not represent temporal information. Many time-frequency analysis techniques have been proposed. However, there is a compromise between time and frequency resolution. The techniques that minimize the EMG noise and analyze signal characteristics are discussed together to identify the best performance with the highest percentage of accuracy and efficiency. The most appropriate method depends on the EMG signal patterns, the quality and quantity of the signals and training data developed, and various types of user factors.
\end{abstract}

KEYWORDS: Digital Signal Processing; Machine Learning; Electromyography

\subsection{INTRODUCTION}

The work on electromyography (EMG) signal processing, as well as the utilization of EMG signal analysis for clinical applications and engineering studies such as prosthetic arms and musculoskeletal disorders, will be discussed in this presentation. The basic theory of myoelectric signal creation will be briefly discussed at the outset of this 
study [1]. Following that, the signal processing techniques used on the EMG signal such as time domain, frequency domain, and the timefrequency domain will be discussed [2].

It includes signal acquisition methods, such as noise removal, as well as signal processing techniques such as amplitude and spectrum analysis. This paper will also look at several works and works of literature on the use of the EMG signal as a tool for various applications such as clinical diagnosis, motion analysis, prosthetic device, and to know the performance of the muscles [3].

Machine learning algorithms, such as traditional machine learning algorithms, and reinforcement learning algorithms, have been widely used in the medical field and have played an important role in the diagnosis and treatment of diseases as an essential component of artificial intelligence [4].

Thus, in this paper, some types of DSP methods and machine learning will be discussed to identify the proper and optimum method that can provide more information from the signal characteristics and the ML to identify the type of algorithm that can give the highest performance comparisons of accuracy.

\subsection{THE RELATION OF DIGITAL SIGNAL PROCESSING, MACHINE LEARNING AND ELECTROMYOGRAPHY}

\subsection{Electromyography (EMG)}

Electromyography (EMG) is the measurement of electrical potential arising from electrochemical effects due to muscle contractions. These signals are transmitted via human tissue to the surface of the skin, where they can be measured by surface EMG electrodes and it is a complicated and non-stationary signal, which is controlled by the human nervous system. Areas of application for these sensors include exoskeletons, diagnostics, and myoelectric hand prostheses [5]-[7]. EMG has become a reliable and cost-effective method for signal acquisition in limbs. Placing electrodes inappropriate position of skin is an important criterion to get the optimum EMG signals [8], [9].

\subsubsection{EMG Signal Analysis}

The EMG signal is a biomedical signal that measures electrical currents generated in muscles during their contraction representing neuromuscular activities. The nervous system continuously controls muscle activity such as during contraction and relaxation. EMG signal is a complex signal, which is controlled by the nervous system and is 
dependent on the anatomical and physiological properties of muscles. EMG signal acquires noise while travelling via different tissues [10]. This signal is a complex and non-stationary signal, which is controlled by the human nervous system. The amplitude of EMG signals is a very small value between $50 \mathrm{uV}$ to $1 \mathrm{mV}$, with the frequencies varying from $10 \mathrm{~Hz}$ to $3000 \mathrm{~Hz}$ [11]. The EMG signals can be measured by applying electrodes to the skin surface (non-invasive method) or intramuscular (invasive method) within the muscle. Even though the efficiency of surface EMG is lower than the intramuscular EMG, but still for research purposes, surface EMG is more popular since it is non-invasive and more convenient to use [12]. Figure 1 shows the EMG signal for normal muscle during contraction.

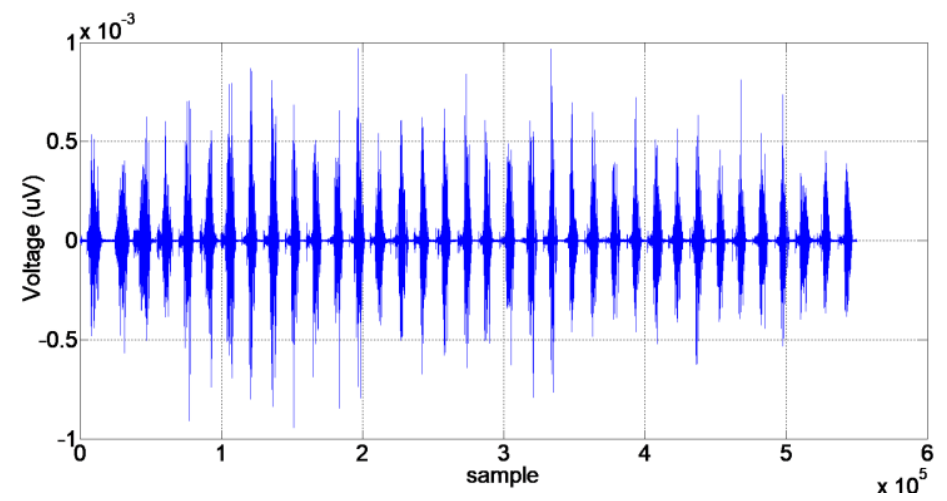

Figure 1: Normal EMG Signal

The flow of EMG signal analysis is divided into some phases for the health screening task. Figure 2 shows the flow phase of EMG signal analysis. There are four phases which are raw data collection of EMG signal, pre-processing, analysis of features extraction and classification of EMG signal by using time-frequency distribution (TFD) and signal classification.

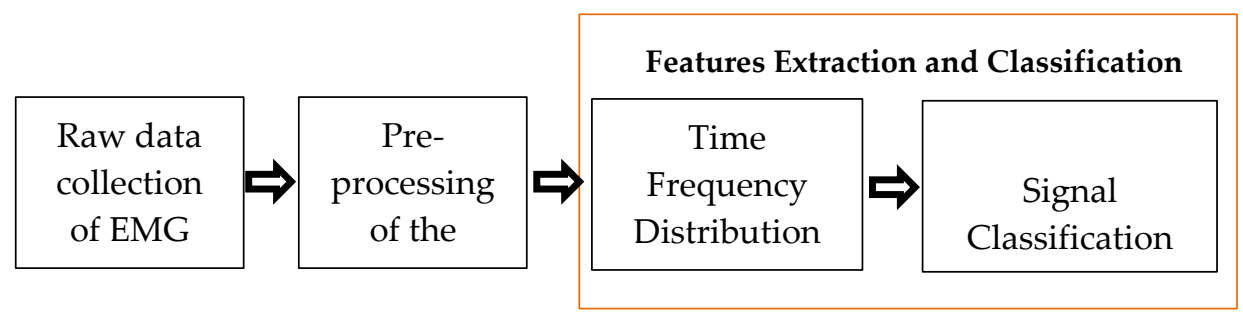

Figure 2: Flow phase of EMG signal analysis

It has been commonly accepted that the preferred manner for 
processing the EMG signal is to calculate the integrated rectified signal. This was done by rectifying (rendering the signal to have excursions of one polarity) the EMG signal, integrating the signal over a specified interval of time, and subsequently forming a time series of the integrated values. The advances made in electronics devices during the past decades have made it possible to conveniently and accurately calculate the root-mean-squared (RMS) and the average rectified (AVR) value of the EMG signal. The AVR value is similar to the integrated rectified value if the calculations are made correctly and accurately.

Both these variables provide a measurement of the area under the signal but do not have a specific physical meaning. The EMG signals which are going to be analyzed are represented in time-frequency representation (TFR) which includes parameters such as instantaneous RMS voltage. Frequency domain analysis that the first frequency analysis technique used is known as the Fast-Fourier Transform (FFT).

FFT is the mathematical technique to convert the signal from a time domain to a frequency domain. FFT is performed to estimate the frequency characteristics of the EMG signal. The limitation of the FFT is that it is not able to cater to non-stationary signals whose spectral characteristic changes in time. This can be overcome by the Linear Time-Frequency distribution (TFD) technique.

To increase the test efficiency of patients' muscle condition a technique to extract information is highly needed. Many timefrequency analysis techniques have been proposed and one of the most commonly used is short-time Fourier transform (STFT) which is a form of linear time-frequency distribution (TFD) [13]. However, the major disadvantage is that it does not represent temporal information. [12]. To solve the problem, Dennis Gabor (1946) improved the Fourier analysis to small sections of signals and divided by the time of analysis in intervals.[14]. The main shortcoming of this method is that there is a compromise between time and frequency resolution. The main disadvantage of this technique is that there is a compromise between time and frequency resolution. The greater the temporal resolution required, the worse the frequency resolution will be and vice versa. Figure 3 shows the example of the time-frequency representation (TFR) of a signal using the STFT. The TFR presents the temporal and spectral information of the signal [15]. 

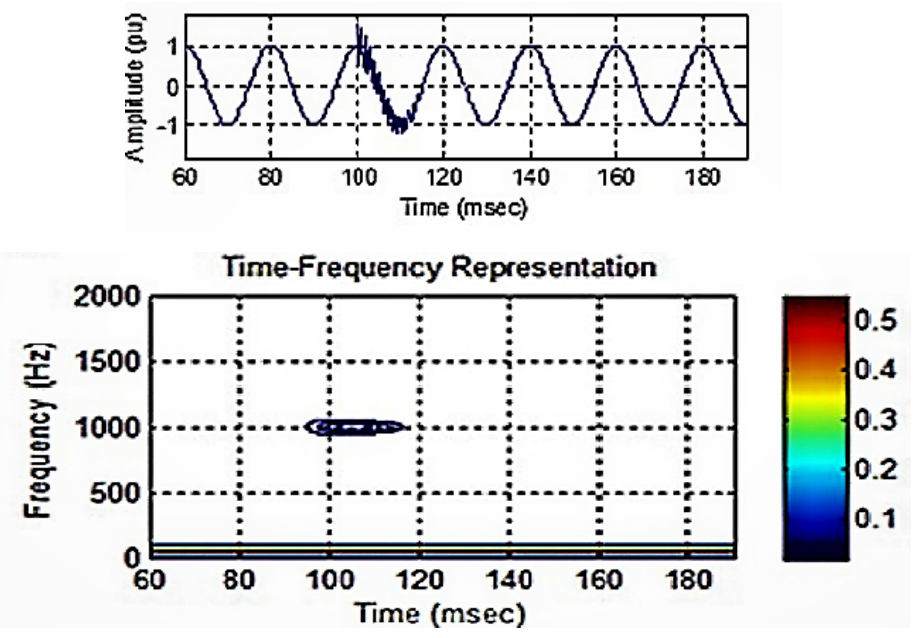

Figure 3: Example of time-frequency representation using Short Time Fourier Transform (STFT) [15]

\subsubsection{Equipment for Data Collection EMG signal}

Consensys is the software that works together with Shimmer sensors which adds significant features to live data, managed data, and devices. It is adaptive human data collection in the field, large-scale repeatable trials, and general multi-sensor management. Consensys enables the following functionalities as Configuring Shimmer sensors, Streaming real-time data, and Managing collected data. Figure $4(\mathrm{a})(\mathrm{b})(\mathrm{c})$ shows the Consensys software Interface [16]. 


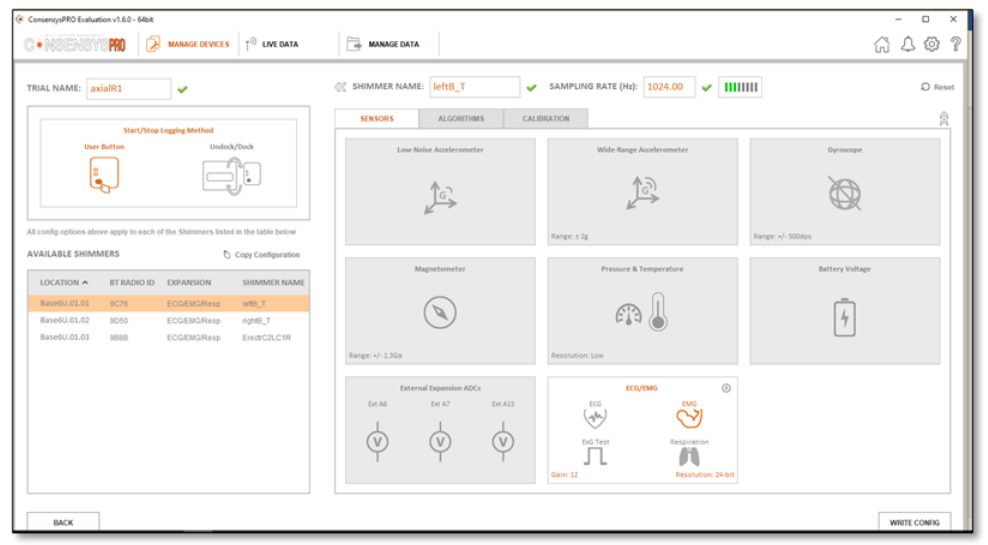

Figure 4: Consensys software Interface [16]

The Shimmer3 Consensys EMG Development Kit can be utilized to monitor two channels of non-invasive surface EMG, providing a representation of the muscle activity at the measurement site. The EMG Development Kit can also be utilized to monitor ECG (Electrocardiogram), recording the pathway of electrical impulses through the heart muscle. Combined with Shimmer's integrated altimeter and 9DoF inertial sensor platform, greater context can be given to the wearer's activity and condition in real-time. Figure 5 shows the Consensys EMG Development Kits [17].

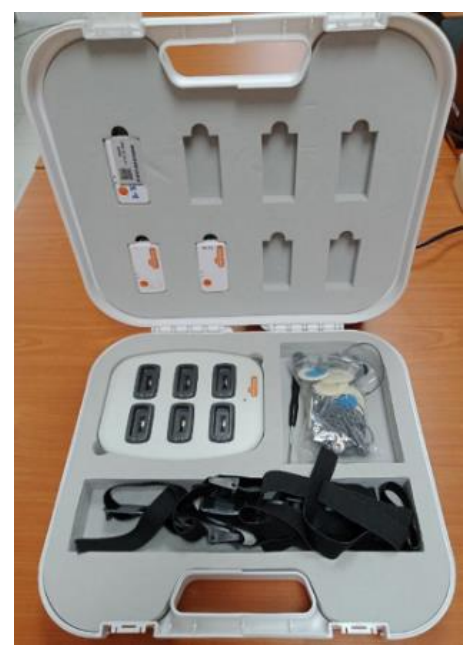

Figure 5: Consensys EMG Development Kits

The Shimmer3 EMG unit can measure and records the electrical activity associated with muscle contractions, assesses nerve conduction, muscle response in injured tissue, activation level, or can 
be used to analyze and measure the biomechanics of human or animal movement. Other than that, it can provide a configurable digital frontend, optimized for the measurement of physiological signals for EMG[18].Shimmer3 EMG sensor consists of two channels of EMG data as shown in Figure 6 and EMG data can be measured simultaneously with 10DOF kinematic data.

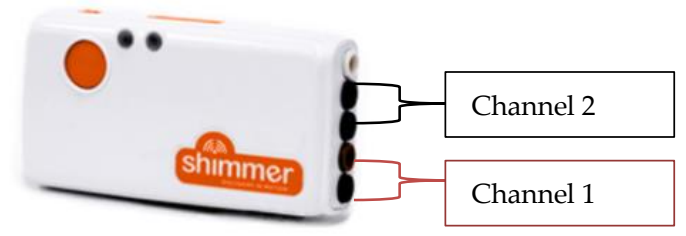

Figure 6: Consensus Shimmer3 EMG Unit

Consensys Base6 (Figure 7) is used to configure and capture data from multiple sensors simultaneously, to simplify the setup of trials and storage of recorded data. The key features of Consensys Base6 consist of Shimmer3 firmware updating, Shimmer configuration, Multi-Shimmer synchronization, and logged data management [19]. Other than that, the functional Base6 is to provide for the simultaneous management of all your Shimmer Sensors including charging, firmware updates, configuring sensors, retrieving and processing logged data. Besides that, Consensys Base6 is compatible with the Shimmer3 range of Kinematic and Biophysical sensors including ECG, EMG, Galvanic Skin Response, Optical Pulse, and Heart Rate as shown in Figure 8.

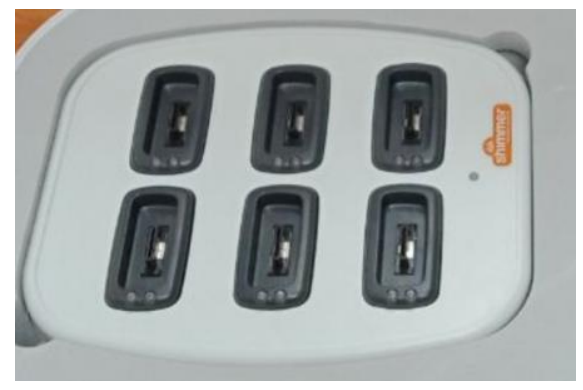

Figure 7: Consensus Shimmer Base6 


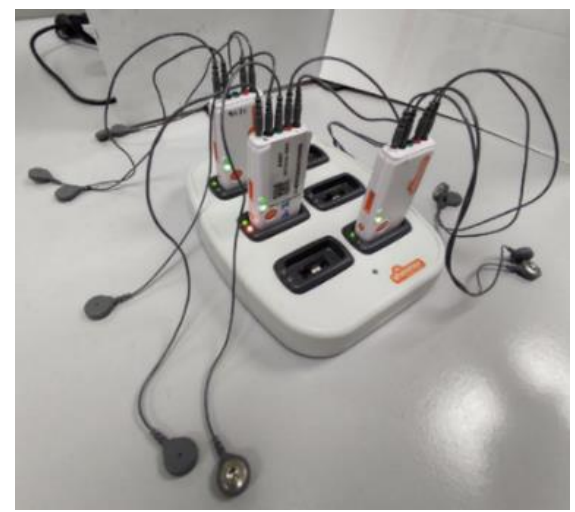

Figure 8: Installation Consensus Shimmer3 EMG Unit on Base6

The Shimmer MATLAB is the Instrument Driver which is an orientated solution for Shimmer to capture the data directly into MATLAB. This driver will allow Shimmer users to stream data directly to MATLAB, and assist users of the Shimmer3 Platform in the development of Shimmer-based applications in MATLAB. Figure 9 shows the interface of the Shimmer MATLAB Instrument Driver [20].

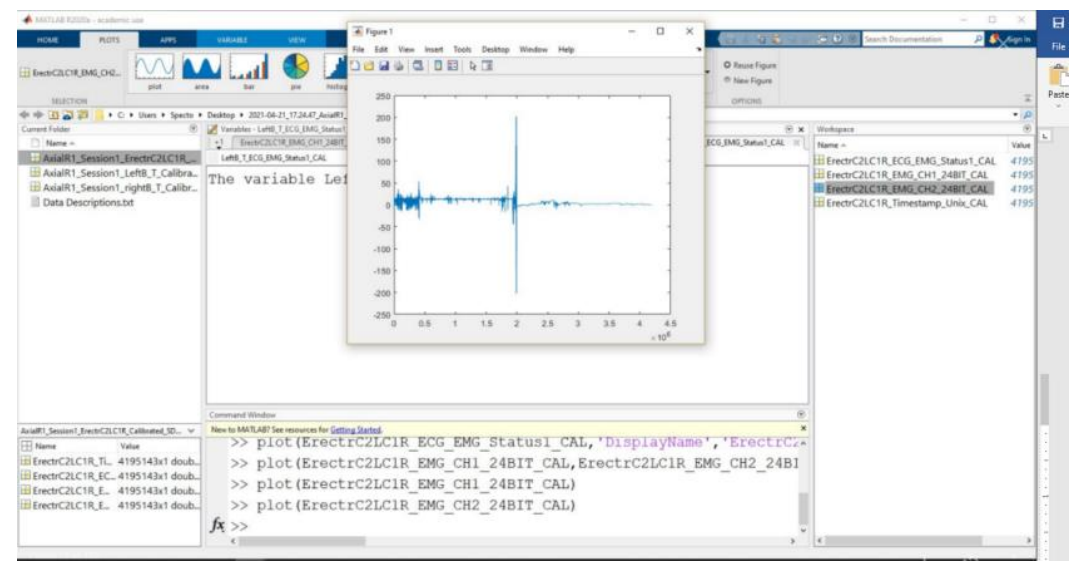

Figure 9: Shimmer MATLAB Instrument Driver [20]

\subsection{Digital Signal Processing (DSP)}

Digital signal processing is known as one of the elementary technologies utilized in the field of biomedical engineering [21] other than communication [22]-[24]. Digital signal processing is the method to process signals by using numerical methods. It is a subject that uses computers or special digital processing equipment. The typical 
purpose of digital signal processing is the acquisition of biological signal, noise reduction, signal transformation, analysis, synthesis, filtering, evaluation, identification, and others so that the required signal can be extracted [1],[25]. The basic flow of the digital signal processing is shown in Figure 10.

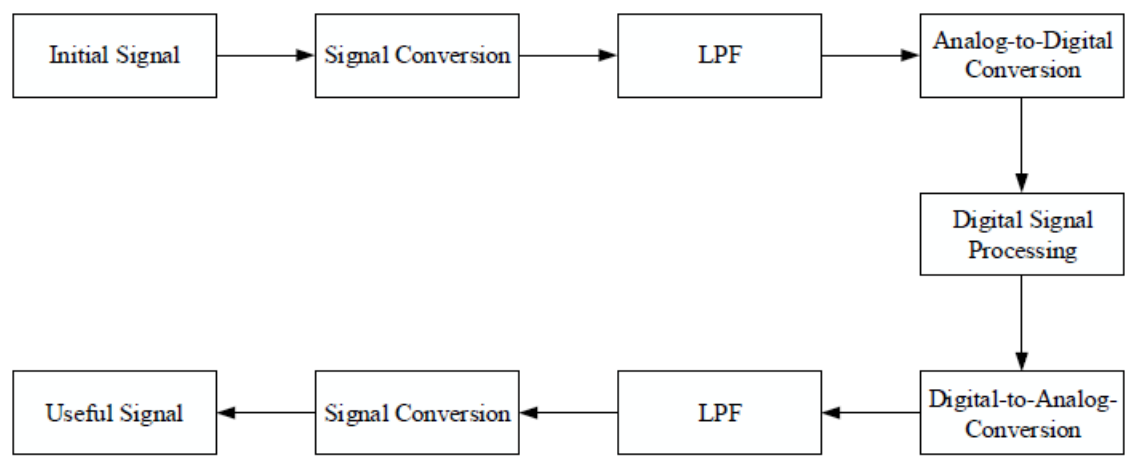

Figure 10: Digital Signal Processing Flow [22]

Digital signal processor that can execute different things, depending on the application being performed. Some of these variants are audio signal processing, audio and video compression, speech processing and recognition, digital image processing, and radar applications. The difference between each of these applications is how the digital signal processor can filter each input. Five different aspects vary from each DSP: clock frequency, RAM size, data bus width, ROM size, and I/O voltage [26].

\subsection{Machine Learning (ML)}

Machine learning is one of the artificial intelligence (AI) that predicts the outcome without relying upon the pre-determined equation. Machine learning learns the information directly from the data and it instructs the computer to do by learning from the experience [17]. Machine learning is divided into supervised and unsupervised learning. More specifically, classification is one type of supervised learning. Unlike unsupervised learning, supervised learning predicts the categorical responses from known response data set [18]. Moreover, machine learning is learned from experience, which is the features extracted from the signals. In recent days, the classifiers such as support vector machines (SVM), k-nearest neighbour (k-NN), linear discriminate analysis (LDA), naïve Bayes (NB), artificial neural network (ANN), general regression neural network (GRNN), multilayer perceptron neural network (MLPNN) and decision tree (DT) are widely used in the 
classification of EMG signals.

Machine learning represents an effective method for data analysis in many domains: it has recently demonstrated its effectiveness in processing tactile sensor data [27]. Machine learning and artificial intelligence applications have grown rapidly across several disciplines, industries, and cultures.[28]-[30].

\subsection{APPLICATION OF DIGITAL SIGNAL PROCESSING AND MACHINE LEARNING FOR EMG}

In 2016 Elhan Umut and Güven Çentik has been studies the Digital Signal Processing in EMG [31] .In this study, the researcher intended to detect periodic leg movement (PLM) in sleep with the use of the channels except for leg electromyography (EMG) by analyzing polysomnography (PSG) data with digital signal processing (DSP) and machine learning methods. PSG records of 153 patients of different ages and genders with PLM disorder diagnoses were examined retrospectively. A novel software was developed for the analysis of PSG records. The software utilizes machine learning algorithms, statistical methods, and DSP methods. To classify PLM, popular machine learning methods (multilayer perceptron, $K$-nearest neighbour, and random forests) and logistic regression were used.

Next, in the same year in 2016, Theresa Roland and his teams used the same application regarding DSP and EMG [32]. The teams introduced the new capacitive measurement system to the application in prostheses. A capacitive EMG prototype, consisting of a flexible sensor and measurement electronics, was developed. The electronic circuit, used for signal amplification and filtering, is described. An ultralow-power microcontroller was used for the implementation of algorithms for EMG signal processing. DSP algorithms were optimized for real-time processing and minimal computing power. Muscle signals, measured with this prototype, are presented.

In the year 2021, Basilio Vescio has developed and validated a new mobile tool for the automated and quantitative characterization of phase displacement resting tremor pattern in ambulatory clinical settings using DSP for $\mu$ EMG application [33]. A new low-cost, wearable mobile device, called, $\mu \mathrm{EMG}$, is described, based on low-end instrumentation amplifiers and simple digital signal processing (DSP) capabilities.

\subsection{Machine Learning in EMG}


Back in 2017, a study has been done by Jianyun that involves ML in EMG studies [34]. In his study, the real-time TMS320C6748 DSP was used instead of Matlab to collect and analyze the surface electromyography (SEMG), to realize the real-time detection of electromyogram (EMG) parameters. He uses the frequency domain feature extraction method to analyze muscle fatigue, and study the effectiveness of the EMG fatigue analysis.

For two years, Jianhua Zhang and his team have developed extracted four time-domain features of the EMG signals and use a generative graphical model, Deep Belief Network (DBN), to classify the EMG signals [35]. A DBN is a fast, greedy deep learning algorithm that can rapidly find a set of optimal weights of a deep network with many hidden layers.

In 2020, similar technologies in ML and EMG have been used in studies done by Yuri Kovalev and JoyRoy [36], [37]. Yuri has created a system for controlling devices using EMG signals from a small number of sensors by recognizing signal sections corresponding to the operator's gestures. The solution implements a scheme of three independent components: sensors, a user interface, and a data processor, connected by a simple data transfer protocol, which allows replacing any of the parts if necessary.

While Joy Roy investigated the different machine learning techniques for predicting the SEMG activities on upper limb muscles. Various issues were presented for predicting SEMG activities on upper limb muscle including muscle classification techniques, muscle selection, and SEMG parameter to signify the muscle activities. These outcomes can be applied for predicting the upper limb muscle activities to identify the critical situation of neuromuscular disorders patients.

Any brain-computer interface (BCI) system must translate signals from the user's brain into messages or commands [38]. Many signal processing and machine learning techniques have been developed for this signal translation. Although these techniques are often illustrated using electroencephalography (EEG) signals, they are also suitable for other brain signals. Gurjit and his team have proposed a novel combination of supervised ML with DSP, resulting in ML-DSP: an alignment-free software tool for ultrafast, accurate, and scalable genome classification at all taxonomic levels [39]. They test ML-DSP by classifying 7396 full mitochondrial genomes at various taxonomic levels, from kingdom to genus, with an average classification accuracy of less than $97 \%$. 


\subsection{CONCLUSION}

From this study, it has been founded that the application of DSP and ML in EMG is widely used through various technologies and implementations. All the studies revealed that DSP and ML are related to each other wherein biosignal processing there is a need of the DSP and ML application into the EMG study to classify the signal to minimize the EMG noise of signal, However, most of the studies do not cover the EMG study using ML and DSP altogether. They only managed to focus on certain areas and segments that are related to the field of their study. Thus, this paper will help the future researcher to find the information on the relationship between EMG, digital signal processing as the technique of signal analysis, and machine learning for classification method to provide the best method and classification for EMG signal or another clinical nerve electrophysiology including electroencephalography (EEG).

\subsection{ACKNOWLEDGMENTS}

The authors would like to thanks the Universiti Teknikal Malaysia Melaka (UTeM), Faculty of Electrical and Electronic Engineering Technology (FTKEE) and Faculty of Electrical Engineering (FKE), Advance Digital Signal Processing (ADSP) Lab, Centre of Robotic and Industrial Automation (CeRIA) and Ministry of Higher Education (MOHE), Malaysia that supported this research under project FRGS/1/2020/FTKEE CERIA/F00428.

\subsection{REFERENCES}

[1] A. K. Mukhopadhyay and S. Samui, "An experimental study on upper limb position invariant EMG signal classification based on deep neural network," Biomed. Signal Process. Control, vol. 55, p. 101669, Jan. 2020.

[2] A. Phinyomark, R. N. Khushaba, and E. Scheme, "Feature extraction and selection for myoelectric control based on wearable EMG sensors," Sensors (Switzerland), vol. 18, no. 5, pp. 1-17, 2018.

[3] A. Norali and M. Som, "Surface Electromyography Signal Processing and Application: A Review," in International Conference on Man-Machine Systems, 2009, no. October, pp. 11-13.

[4] T. Liu, Z. Li, Y. Tang, D. Yang, S. Jin, and J. Guan, “The application of the machine learning method in electromyographic data," IEEE 
Access, vol. 8, pp. 9196-9208, 2020.

[5] T. Roland, S. Amsuess, M. F. Russold, and W. Baumgartner, "Ultralow-power digital filtering for insulated EMG sensing," Sensors (Switzerland), vol. 19, no. 4, p. 959, 2019.

[6] M. A. Cavalcanti Garcia and T. M. M. Vieira, "Surface electromyography: Why, when and how to use it," Rev. Andaluza Med. del Deport., vol. 4, no. 1, pp. 17-28, 2011.

[7] "Prosthetics 2017/18, Upper Limb, Otto Bock Healthcare GmbH." https://www.ottobock.se/media/local_media_1/bu-

prosthetics/nedladdning/ottobock__prosthetics__ul_catalogue_64 6k6_gb_04_1709.pdf (accessed May 29, 2021).

[8] K. Samarawickrama, S. Ranasinghe, Y. Wickramasinghe, W. Mallehevidana, V. Marasinghe, and K. Wijesinghe, "Surface EMG Signal Acquisition Analysis and Classification for the Operation of a Prosthetic Limb," Int. J. Biosci. Biochem. Bioinforma., vol. 8, no. 1, pp. 32-41, 2018.

[9] Y. Blanc and U. Dimanico, "Electrode Placement in Surface Electromyography (sEMG) "Minimal Crosstalk Area" (MCA)," Open Rehabil. J., vol. 3, no. 1, pp. 110-126, 2014.

[10] R. H. Chowdhury, M. B. I. Reaz, M. A. Bin Mohd Ali, A. A. A. Bakar, K. Chellappan, and T. G. Chang, "Surface electromyography signal processing and classification techniques," Sensors (Switzerland). 2013.

[11] B. Widanarko et al., "Prevalence and work-related risk factors for reduced activities and absenteeism due to low back symptoms," Appl. Ergon., vol. 43, no. 4, pp. 727-737, Jul. 2012.

[12] A. Costa, M. Itkonen, H. Yamasaki, F. S. Alnajjar, and S. Shimoda, "Importance of muscle selection for EMG signal analysis during upper limb rehabilitation of stroke patients," 2017.

[13] H. Daneshmandi, A. R. Choobineh, H. Ghaem, M. Alhamd, and A. Fakherpour, "The effect of musculoskeletal problems on fatigue and productivity of office personnel: A cross-sectional study," J. Prev. Med. Hyg., vol. 58, no. 3, pp. E252-E258, 2017.

[14] D. Starovoytova, "Hazards and Risks at Rotary Screen Printing (Part 2/6): Analysis of Machine-operators' Posture via Rapid-Upper-LimbAssessment (RULA)," Ind. Eng. Lett., vol. 7, no. 5, pp. 42-63, 2017.

[15] B. Boashash, Time-Frequency Signal Analysis and Processing: A Comprehensive Reference. Oxford, 2003.

[16] "ConsensysPRO Software I Collect and analyze biometric and motion data from Shimmer sensors." https://www.shimmersensing.com/products/consensys. 
[17] "Consensys Bundle Development kit I Complete Wearable Sensor Kit I IMU - ECG - EMG - GSR." https://www.shimmersensing.com/products/consensys-ecgdevelopment-kits-update.

[18] "Wearable EMG Sensor I Wireless EMG sensor I Electromyogram." https://www.shimmersensing.com/products/shimmer3-emgsensor.

[19] "Physiological \& kinematic data capture with the Base6 I Multi sensor management | Charging dock." https://www.shimmersensing.com/products/consensys-base6.

[20] "Shimmer MATLAB Instrument Driver - File Exchange - MATLAB Central."

https://ww2.mathworks.cn/matlabcentral/fileexchange/43712shimmer-matlab-instrument-driver.

[21] Y. Ono, "Back to basics series: Digital signal processing," Trans. Japanese Soc. Med. Biol. Eng., vol. 57, no. (2-3), pp. 75-80, 2019.

[22] H. Lu, Y. Xiaoyu, W. Haodong, L. Jin, M. Xuejiao, and Z. Caihong, "Research on Application of Digital Signal Processing Technology in Communication," 2020.

[23] K. Zhong, X. Zhou, J. Huo, C. Yu, C. Lu, and A. P. T. Lau, “Digital Signal Processing for Short-Reach Optical Communications: A Review of Current Technologies and Future Trends," J. Light. Technol., vol. 36, no. 2, pp. 1-24, 2018.

[24] T. Kobayashi, F. Hamaoka, M. Nakamura, H. Yamazaki, M. Nagatani, and Y. Miyamoto, "Ultrahigh-speed optical communications technology combining digital signal processing and circuit technology," NTT Tech. Rev., 2019.

[25] D. Goyal, C. Mongia, and S. Sehgal, "Applications of Digital Signal Processing in Monitoring Machining Processes and Rotary Components: A Review," IEEE Sensors Journal, vol. 21, no. 7. 2021.

[26] D. Krambeck, "An Introduction to Digital Signal Processing Technical Articles," 2015. https://www.allaboutcircuits.com/technical-articles/anintroduction-to-digital-signal-processing/ (accessed May 29, 2021).

[27] A. Ibrahim, P. Gastaldo, H. Chible, and M. Valle, "Real-time digital signal processing based on FPGAs for electronic skin implementation," Sensors (Switzerland), vol. 17, no. 558, p. 558, 2017.

[28] U. S. Shanthamallu, S. Rao, A. Dixit, V. S. Narayanaswamy, J. Fan, and A. Spanias, "Introducing Machine Learning in Undergraduate DSP Classes," 2019. 
[29] M. Kandlhofer, G. Steinbauer, S. Hirschmugl-Gaisch, and P. Huber, "Artificial intelligence and computer science in education: From Kindergarten to university," 2016.

[30] T. Barik, M. Everett, R. E. Cardona-Rivera, D. L. Roberts, and E. F. Gehringer, "A community college blended learning classroom experience through Artificial Intelligence in Games," 2013.

[31] I. Umut and G. Çentik, "Detection of Periodic Leg Movements by Machine Learning Methods Using Polysomnographic Parameters Other Than Leg Electromyography," Comput. Math. Methods Med., vol. 2016, no. 1, pp. 1-7, 2016.

[32] T. Roland, W. Baumgartner, S. Amsuess, and M. F. Russold, "Signal evaluation of capacitive EMG for upper limb prostheses control using an ultra-low-power microcontroller," in IECBES 2016 - IEEEEMBS Conference on Biomedical Engineering and Sciences, 2016, pp. 317-320.

[33] B. Vescio, R. Nisticò, A. Augimeri, A. Quattrone, M. Crasà, and A. Quattrone, "Development and Validation of a New Wearable Mobile Device for the Automated Detection of Resting Tremor in Parkinson's Disease and Essential Tremor," Diagnostics, vol. 11, no. 2, p. 200, 2021.

[34] J. Han, "Application of EMG fatigue detection algorithm in portable DSP system," Acta Tech. CSAV (Ceskoslovensk Akad. Ved), vol. 62, no. 3, pp. 85-94, 2017.

[35] J. Zhang, C. Ling, and S. Li, "Human movements classification using multi-channel surface EMG signals and deep learning technique," in Proceedings - 2019 International Conference on Cyberworlds, CW 2019, Oct. 2019, pp. 267-273.

[36] Y. Kovalev, T. Bergaliyev, and S. Sakhno, "An Interactive System for the Study of EMG Signal ML-Processing and Prototyping of HumanMachine Interfaces," in 2020 International Conference Engineering and Telecommunication (EnET), Nov. 2020, pp. 1-3, Accessed: May 30, 2021. [Online]. Available: https://ieeexplore.ieee.org/document/9431317/.

[37] J. Roy, M. A. Ali, M. R. Ahmed, and K. Sundaraj, “Machine learning techniques for predicting surface EMG activities on upper limb muscle: A systematic review," in Lecture Notes of the Institute for Computer Sciences, Social-Informatics and Telecommunications Engineering, LNICST, 2020, pp. 330-339.

[38] Y. Li, K. K. Ang, and C. Guan, "Digital Signal Processing and Machine Learning," in Brain-Computer Interfaces. The Frontiers Collection., Graimann B.; Pfurtscheller G.; and Allison B., Eds. 
Springer, Berlin, Heidelberg, 2009, pp. 305-330.

[39] G. S. Randhawa, K. A. Hill, and L. Kari, “ML-DSP: Machine Learning with Digital Signal Processing for ultrafast, accurate, and scalable genome classification at all taxonomic levels," BMC Genomics, vol. 20, no. 1, 2019. 